\title{
The Muon Anomalous Magnetic Moment
}

\author{
Marc Knecht* \\ Centre de Physique Théorique, CNRS/Aix-Marseille Univ./Univ. du Sud Toulon-Var (UMR 7332) \\ CNRS-Luminy Case 90\%, 13288 Marseille Cedex 9, France
}

\begin{abstract}
The calculations entering the prediction of the standard model value for the anomalous magnetic moment of the muon $a_{\mu}$ are reviewed, and compared to the very accurate experimental measurement. The situation for the electron is discussed in parallel.
\end{abstract}

\section{INTRODUCTION}

The Dirac equation, together with minimal coupling to an external electromagnetic field, predicts, for an elementary charged lepton $\ell$ of spin $1 / 2$, mass $m_{\ell}$, and charge $e_{\ell}$, a Pauli-type coupling $\boldsymbol{\mu}_{\boldsymbol{\ell}} \cdot \mathbf{B}$ of the spin to the magnetic field, where the magnetic moment, proportional to the particle's spin, is given in units of the Bohr magneton, by

$$
\boldsymbol{\mu}_{\ell}=g_{\ell}^{\text {Dirac }}\left(\frac{e_{\ell}}{2 m_{\ell} c}\right) \hbar \frac{\sigma}{2}
$$

with $g_{\ell}^{\text {Dirac }}=2$. Quantum corrections arising from loops will bring in new contributions and shift the gyromagnetic factor $g_{\ell}$ away from this value. Given a sufficient level of precision, all degrees of freedom, known or unknown (i.e. light or heavy), will eventually contribute in a visible way. The existence of an anomalous magnetic moment $a_{\ell} \equiv\left(g_{\ell}-g_{\ell}^{\text {Dirac }}\right) / g_{\ell}^{\text {Dirac }}$ is thus an indirect probe of the existence and properties of all degrees of freedom, within, but also beyond, the standard model.

It actually turns out that, on the experimental level, the anomalous magnetic moments of the electron $\left(a_{e}\right)$ and of the muon $\left(a_{\mu}\right)$ are among the most precisely measured low-energy observables in particle physics. In the case of the muon, the very precisely known experimental value [1]

$$
a_{\mu}^{\exp }=11659208.9(6.3) \cdot 10^{-10} \quad[0.54 \mathrm{ppm}],
$$

is dominated by the results obtained by the BNL-E821 experiment [2, 3]. The situation is even more impressive in the case of the electron. The value

$$
a_{e}^{\exp }=1159652180.73(0.28) \cdot 10^{-12}[0.24 \mathrm{ppb}]
$$

follows from the measurement, at a relative precision of $0.28 \mathrm{ppt}$, of the gyromagnetic factor $g_{e}[4]$.

The high level of precision achieved by these experiments is certainly challenging for theory and theoreticians. The question naturally arises, whether theoretical evaluations are able to reach the same level of accuracy, and whether the standard model accounts for these values. Most exciting, of course, is the possibility that eventually a significant discrepancy (with reliable theoretical and experimental uncertainties) remains, thus signaling the existence of degrees of freedom beyond the standard model.

This review summarizes the present status of the theoretical evaluations of $a_{\mu}$ and of $a_{e}$. In order to reach an accuracy comparable to the experimental results, it is necessary to consider contributions from electromagnetic interactions (section 2), from weak interactions (section 3), and from strong interactions (section 4). The latter, although larger than the corrections from the standard model weak interactions, are particularly challenging, since they heavily involve the non-perturbative regime. This is why they have been kept for the end. This review will end with Section 5 , which includes a summary and some conclusions, and presents perspectives for the future.

\section{QED CONTRIBUTIONS}

The interactions involving only photons and charged leptons can be treated perturbatively,

*Electronic address: knecht@cpt.univ-mrs.fr 
TABLE I: The coefficients $C_{\ell}^{(2 n)}$, of the perturbative QED expansion for the anomalous magnetic moment of the electron (left) and of the muon (right). The values are taken from [8] and [9].

\begin{tabular}{|c|c|c|}
\hline & $\ell=e$ & $\ell=\mu$ \\
\hline$C_{\ell}^{(2)}$ & 0.5 & 0.5 \\
$C_{\ell}^{(4)}$ & $-0.32847844400 \ldots$ & $0.765857425(17)$ \\
$C_{\ell}^{(6)}$ & $1.181234017 \ldots$ & $24.05050996(32)$ \\
$C_{\ell}^{(8)}$ & $-1.9096(20)$ & $130.8796(63)$ \\
$C_{\ell}^{(10)}$ & $9.16(58)$ & $753.29(1.04)$ \\
\hline \multicolumn{2}{|c|}{$a_{\ell}^{\mathrm{QED}}=\sum_{n \geq 1} C_{\ell}^{(2 n)}\left(\frac{\alpha}{\pi}\right)^{n}}$.
\end{tabular}

The challenge comes, however, from the high orders in the perturbative expansion that one needs to consider in order to reach a level of precision comparable to the experimental one. In the case of the electron, the experimenal uncertainty $\Delta a_{e}^{\exp }=2.8 \cdot 10^{-13}$, together with $(\alpha / \pi)^{4} \sim 3 \cdot 10^{-11}$, indicates that one needs to compute at least five loop contributions. The one-loop coefficient $C_{e}^{(2)}=C_{\mu}^{(2)}=1 / 2$ was obtained by J. Schwinger [5] long ago. The two-loop and three-loop coefficients are also known analytically. For surveys of these calculations, and references to the original works see [6]. The higher order coefficients involve 891 diagrams at four loops, and 12672 diagrams at five loops. They have been computed through systematic numerical evaluations of the multidimensional integrals over the corresponding Feynman parameters. Complete results have now been published [7-9]. The results of these calculations are displayed in Table I, and we merely make a few comments.

i) The accuracy of the coefficients $C_{\mu}^{(4)}$ and $C_{\mu}^{(6)}$, which are known analytically, is actually limited by the uncertainties on the experimental values [10] of the mass ratios $m_{\mu} / m_{e}$ and, to a lesser extent, $m_{\mu} / m_{\tau}$ and $m_{e} / m_{\tau}$. In the electron case, these uncertainties would only affect the digits beyond those shown, and are not relevant given the present size of $\Delta a_{e}^{\exp }$. The uncertainties on the four and five-loop coefficients come, in both cases, from the numerical integration procedure.

ii) In the muon case, the experimental accuracy is somewhat lower, $\Delta a_{\mu}^{\exp }=6.3 \cdot 10^{-10}$, and one might think that even the fourth order is not needed. But this is without reckoning with the structure of the coefficients, which, in the muon case, are logarithmically enhanced by the presence of the much lighter electron in the loops. Starting at three loops, one encounters terms involving $\pi^{2} \ln \left(m_{\mu}^{2} / m_{e}^{2}\right) \sim 50$ ! This explains why the coefficients $C_{\mu}^{(2 n)}$ in Table I are typically larger than in the electron case. Despite the size of $C_{\mu}^{(10)}, C_{\mu}^{(10)}(\alpha / \pi)^{5} \sim 0.5 \cdot 10^{-10}$, so that this contribution remains marginal in view of the present experimental error. But it will also have to be considered as the latter improves (see Section 5).

iii) Some subsets of diagrams contributing to $C_{\ell}^{(8)}$ and $C_{\ell}^{(10)}$ are also known analytically [11 15], in terms of expansions in powers of the mass ratios. These analytical results provide useful and welcome checks of the numerical evaluations. iv) Finally, notice that $\Delta C_{\mu}^{(2 n)}(\alpha / \pi)^{n}$, where $\Delta C_{\mu}^{(2 n)}$ denotes the uncertainty on $C_{\mu}^{(2 n)}$, is below the experimental precision $\Delta a_{\mu}^{\exp }=6.3 \cdot 10^{-10}$ for $n=2,3,4,5$, so that in practice $a_{\mu}^{\mathrm{QED}}$ is, for the time being, free of any theoretical error.

At this stage, using the latest high-precision measurement of the fine-structure constant [16]

$$
\alpha^{-1}=137.035999037(91) \quad[0.66 \mathrm{ppb}]
$$

we have

$$
a_{e}^{\mathrm{QED}}=1159652180.07(6)(4)(77) \cdot 10^{-12}
$$

and

$$
a_{\mu}^{\mathrm{QED}}=1165847189.51(19)(7)(77)(9) \cdot 10^{-12} .
$$

The first two errors come from the contributions at orders $\mathcal{O}\left(\alpha^{4}\right)$ and $\mathcal{O}\left(\alpha^{5}\right)$, respectively, and the third one comes from the experimental uncertainty on $\alpha$. In the case of $a_{\mu}^{\mathrm{QED}}$ there is an additional error from the uncertainties on the mass ratios. It then follows that

$$
\begin{aligned}
& a_{e}^{\exp }-a_{e}^{\mathrm{QED}}=+0.67(82) \cdot 10^{-12} \\
& a_{\mu}^{\exp }-a_{\mu}^{\mathrm{QED}}=+737.0(6.3) \cdot 10^{-10}
\end{aligned}
$$


In the case of the electron, $a_{e}$ is well described by QED, within the uncertainties, which are dominated by the present uncertainty on the determination of $\alpha$. In the case of the muon, the discrepancy with the experimental value is substantial, so that the difference is to be ascribed to the contributions of the weak and of the strong interactions.

Notice that one can use the measurement of $a_{e}$ in order to reduce (by a factor 2.5) the error on $\alpha$ and obtain a determination of the fine-structure constant at the $0.25 \mathrm{ppb}$ level [8]. But this means that $a_{e}$ then no longer provides a possible test of the standard model (more on this at the end of Section 5).

\section{CONTRIBUTIONS FROM THE WEAK INTERACTIONS}

The one-loop contributions due to the weak interaction sector of the standard model have been computed [17 21] more than forty years ago. They read

$$
a_{\ell}^{\text {weak }(1)}=\frac{G_{F}}{\sqrt{2}} \frac{m_{\mu}^{2}}{8 \pi^{2}}\left[\frac{5}{3}+\frac{1}{3}\left(1-4 \sin ^{2} \theta_{W}\right)^{2}+\mathcal{O}\left(\frac{m_{\mu}^{2}}{M_{Z}^{2}} \log \frac{M_{Z}^{2}}{m_{\mu}^{2}}\right)+\mathcal{O}\left(\frac{m_{\mu}^{2}}{M_{H}^{2}} \log \frac{M_{H}^{2}}{m_{\mu}^{2}}\right)\right],
$$

and correspond numerically to $a_{\mu}^{\text {weak(1)}}=19.48 \cdot 10^{-10}$. Two-loop electroweak corrections are also available [22 24], and reduce the above value by about 20\%. A recent reevaluation [25] gives, for the sum of the one- and two-loop weak contributions,

$$
\begin{aligned}
& a_{\mu}^{\text {weak }}=+15.4(1) \cdot 10^{-10} \\
& a_{e}^{\text {weak }}=+0.297(5) \cdot 10^{-13}
\end{aligned}
$$

After taking these contributions into account, we now have

$$
a_{\mu}^{\mathrm{exp}}-a_{\mu}^{\mathrm{QED}}-a_{\mu}^{\mathrm{weak}}=+721.65(6.38) \cdot 10^{-10}
$$

and only the strong interactions remain in order to close this gap.

\section{CONTRIBUTIONS FROM THE STRONG INTERACTIONS}

The strong-interaction contributions mainly come from the low-energy, light-quark, sector, so that a perturbative approach is no longer adapted. It is both useful and customary to distinguish three types of corrections involving the hadronic sector, as shown in Fig. 1. The diagram on the left corresponds to hadronic contributions to the photon vacuum polarization function. The diagram in the middle is the so-called hadronic light-by-light contribution. Finally, there is also a hadronic contribution to the two-loop weak corrections discussed in the previous section, arising from the exchange of a virtual photon and a virtual neutral weak gauge boson between the external lepton line and the hadronic blob (right diagram). In comparison to the other two-loop weak corrections, this last correction is small. It was already included in the values given in the preceding section, and will not be discussed further here. For details, I refer the reader to the literature quoted in the preceding section.

\section{A. Hadronic vacuum polarization}

Let us start by examining the case of hadronic vacuum polarization. This contribution can be expressed as [26 28

$$
a_{\ell}^{\mathrm{HVP}-\mathrm{LO}}=\frac{1}{3}\left(\frac{\alpha}{\pi}\right)^{2} \int_{4 M_{\pi}^{2}}^{\infty} \frac{d s}{s} K(s) R^{\mathrm{had}}(s)
$$

with

$$
K(s)=\int_{0}^{1} d x \frac{x^{2}(1-x)}{x^{2}+(1-x) \frac{s}{m_{\ell}^{2}}}
$$

and $R^{\text {had }}(s)$ represents the $R$-ratio of the cross section of $e^{+} e^{-} \rightarrow$ hadrons. The advantage of this representation is threefold. First it tells us that the contribution is positive $(K(s)>0)$. Second, that it is dominated by the lowenergy domain, since $K(s) \propto m_{\ell}^{2} / s$ for $s$ large. And finally, it is directly related to an experimental quantity, which 


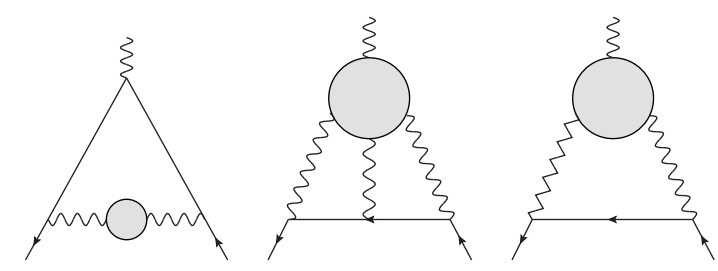

FIG. 1: Hadronic contributions to the anomalous magnetic moment of a charged lepton. One distinguishes hadronic vacuum polarization insertion (left), involving the $\langle V V\rangle$ two-point function, hadronic light-by-light scattering (middle), involving the $\langle V V V V\rangle$ four-point function, and a hadronic contribution to the two-loop weak corrections (right), involving the $\langle V V A\rangle$ threepoint function. Here $V$ stands for the hadronic part of the electromagnetic current, and $A$ denotes the axial part of the hadronic neutral current. In the last two cases, only one typical diagram (out of 6 and 2, respectively) is displayed. At the perturbative level, the blob would correspond to a quark loop, dressed with additional virtual gluons and quarks.

allows $a_{\ell}^{\mathrm{HVP}-\mathrm{LO}}$ to be evaluated using available data on $e^{+} e^{-} \rightarrow$ hadrons. The results from the two latest published evaluations [29, 30] give comparable values,

$$
\begin{array}{lll}
a_{\mu}^{\mathrm{HVP}-\mathrm{LO}}=+692.3(4.2) \cdot 10^{-10} & {[29],} \\
a_{\mu}^{\mathrm{HVP}-\mathrm{LO}}=+694.9(4.3) \cdot 10^{-10} & {[30] .}
\end{array}
$$

The corresponding value for the electron reads 31

$$
a_{e}^{\mathrm{HVP}-\mathrm{LO}}=+1.866(11) \cdot 10^{-12},
$$

and is larger than the present uncertainty on $a_{e}^{\mathrm{QED}}$.

There are also contributions of the same type at order $\mathcal{O}\left(\alpha^{3}\right)$, obtained upon inserting a second (hadronic or leptonic) vacuum polarization in one of the two virtual photons of the leftmost diagram in Fig. 1 or upon inserting a hadronic vacuum polarization in one of the photon lines of the two-loop QED contributions. These contributions can be written in a similar form as in Eq. (IV.12), but involve now a (known) kernel different from $K(s)$ [32, 33]. These next-to-leading hadronic vacuum polarization corrections, evaluated with the same $e^{+} e^{-} \rightarrow$ hadrons data, amount to [30]

$$
a_{\mu}^{\mathrm{HVP}-\mathrm{NLO}}=-9.84(7) \cdot 10^{-10}
$$

for the muon, and [31] $a_{e}^{\mathrm{HVP}-\mathrm{NLO}}=-2.234(14) \cdot 10^{-13}$ for the electron. Recently, the next-next-to-leading hadronic vacuum polarization corrections were also evaluated [34], with the result

$$
a_{\mu}^{\mathrm{HVP}-\mathrm{NNLO}}=+1.24(1) \cdot 10^{-10} .
$$

Summing these three contributions gives $a_{\mu}^{\mathrm{HVP}}=+683.7(4.2) \cdot 10^{-10}$ with the input from [29], or $a_{\mu}^{\mathrm{HVP}}=$ $+686.3(4.3) \cdot 10^{-10}$ with the input from [30], and the difference $a_{\mu}^{\exp }-a_{\mu}^{\mathrm{QED}}-a_{\mu}^{\text {weak }}-a_{\mu}^{\mathrm{HVP}}$ equals $+37.95(7.64) \cdot 10^{-10}$ or $+35.35(7.69) \cdot 10^{-10}$, respectively.

\section{B. Hadronic light-by-light scattering}

We have kept for the end the hadronic contribution to virtual light-by-light scattering, that remains the most challenging one at present. Here, we have no direct link to an experimental observable, and even the overall sign cannot be fixed from the outset. One has to resort to a certain amount of model dependence in order to describe, in the non-perturbative regime, the hadronic four-point function (see Fig. 1) that is involved. Only two calculations have tried to give a complete description of this four-point function from the point of view of its contribution to $a_{\mu}$. After correcting for the sign [35] of the dominant contribution coming from the reducible exchange of a single pseudoscalar meson between pairs of (virtual) photons, their results [36, 37] read

$$
\begin{aligned}
& a_{\mu}^{\mathrm{HLxL}}=+8.3(3.2) \cdot 10^{-10} \quad[36], \\
& a_{\mu}^{\mathrm{HLxL}}=+8.96(1.54) \cdot 10^{-10} \quad[37],
\end{aligned}
$$


and show good agreement. But if one looks into the details 38] of these calculations, there are sometimes sizeable differences, either between contributions common to the two models, or in the contributions that are included or not. The limit of a large number of colours $N_{c}$ [39, 40] can be useful in order to organize the various contributions [41]. At leading order, one has to consider contributions from reducible single meson exchanges (pseudoscalars, axial vectors, tensors). Of these, those of higher masses will have their contributions suppressed, so that the contribution from single $\pi^{0}$ exchanges dominates. This is indeed the case in the models that have been considered in [36, 37]. In the framework of the effective low-energy theory, the leading-order contribution can be worked out exactly [42],

$$
a_{\mu}^{\mathrm{HLxL} ; \pi^{0}}=N_{c}\left(\frac{\alpha}{\pi}\right)^{3} \frac{N_{c}}{F_{\pi}^{2}} \frac{m_{\mu}^{2}}{48 \pi^{2}}\left[\ln ^{2} \frac{M_{\rho}}{M_{\pi}}+c_{\chi} \ln \frac{M_{\rho}}{M_{\pi}}+\kappa\right] .
$$

In this expression, $F_{\pi}$ is the pion-decay constant $\left(F_{\pi} \sim \sqrt{N_{c}}\right.$ in the large- $N_{c}$ limit $)$, and $M_{\rho}$, the mass of the $\rho$ meson, is a typical hadronic mass scale where the effective theory ceases to be applicable. The constant $c_{\chi}$ in front of the subleading contribution is related [42, 43] to a low-energy constant $\chi$ [44] that also appears in the decay $\pi^{0} \rightarrow e^{+} e^{-}$, and can thus be extracted [45] from data. The last constant, $\kappa$, is not fixed by any requirement. In (IV.19), the sign of the leading contribution comes out as positive. Since it results from a model-independent approach, this result has a general validity. In model calculations, the scale $M_{\rho}$ enters through the form factors that describe the $\pi^{0}-\gamma^{*}-\gamma^{*}$ vertex, and which are necessary in order to make the contribution from $\pi^{0}$ exchange finite (taking the constant vertex that follows from the Wess-Zumino term would lead to a divergent contribution). As the UV-regulator $M_{\rho}$ is sent to infinity, one should recover the behaviour (IV.19). This has been checked in a variety of models [35].

Although the pion-exchange practically gives the final result in [36, 37], this partly also results from the cancellation among other contributions, which, taken individually, can be sizeable. Some of these contributions are regularly updated or reevaluated (for a recent example see [46], and the contributions in [47]). There are also contributions coming from short distances, and an important issue is the matching between contributions arising from different scales and regimes. This is certainly a feature that one should improve. For instance, although some short-distance constraints are implemented [36, 48, 49], an extensive study of the four-point function (cf. Fig. 1) from this point of view has not been done so far. Eventually, the total error on $a_{\mu}^{\mathrm{HLxL}}$ will not only reflect our ability to reduce the errors on individual contributions, but must also reflect our confidence in the way we are able to put them together, while respecting the known properties of QCD at short and at long distances.

In order to summarize the present situation, one may quote the "best estimate" from [50]

$$
a_{\mu}^{\mathrm{HLxL}}=10.5(2.6) \cdot 10^{-10},
$$

whereas a more conservative estimate [51] gives

$$
a_{\mu}^{\mathrm{HLxL}}=11.5(4.0) \cdot 10^{-10} .
$$

In the case of the electron, this contribution is much smaller, and one finds [50]

$$
a_{e}^{\mathrm{HLxL}}=0.035(10) \cdot 10^{-12}
$$

\section{SUMMARY, CONCLUSIONS, PERSPECTIVES}

The anomalous magnetic moments of the electron and of the muon are among the most precisely measured lowenergy observables of the standard model. The standard-model value obtained for $a_{e}$ agrees with the experimental measurement

$$
a_{e}^{\exp }-a_{e}^{\mathrm{SM}}=-1.04(82) \cdot 10^{-12}
$$

However, at present the value obtained for $a_{\mu}$ wihin the standard model misses the experimental one. The difference

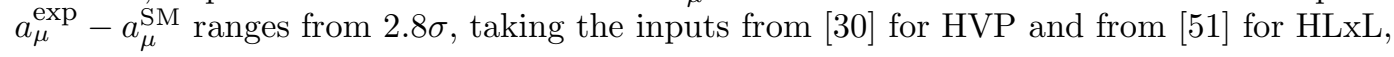

$$
a_{\mu}^{\exp }-a_{\mu}^{\mathrm{SM}}=23.7(8.6) \cdot 10^{-10} \quad[30,51]
$$

to $3.4 \sigma$,

$$
a_{\mu}^{\exp }-a_{\mu}^{\mathrm{SM}}=27.4(8.0) \cdot 10^{-10} \quad[29,50]
$$

when the inputs for HVP and HLxL are taken from [29] and [50], respectively. This discrepancy has been with us for quite some time, and it is not obvious to find a straightforward explanation for it. It is almost twice as large as the 
correction from the weak interactions (Section 3), and the evaluation of the hadronic light-by-light correction would have to be off by a factor of 2 to 3 to explain it.

Could it come from higher order QED effects? After all, the coefficients $C_{\mu}^{(2 n)}$ in Table 1 display a dramatic increase with $n$. An estimate of the contribution at twelfth order $A_{2}^{(12)}\left(m_{\mu} / m_{e}\right)$ that shows the enhancement mechanism mentioned previously, based on the electron ligh-by-light loop $A_{2}^{(6)}\left(m_{\mu} / m_{e} ; \mathrm{LxL}\right)$, corrected by three electron loops, inserted in all possible ways in the three photon lines that are internal to the diagram, gives [9]

$$
\begin{aligned}
A_{2}^{(12)}\left(m_{\mu} / m_{e}\right) & \sim A_{2}^{(6)}\left(m_{\mu} / m_{e} ; \mathrm{LxL}\right)\left[\frac{2}{3} \ln \frac{m_{\mu}}{m_{e}}-\frac{5}{9}\right]^{3} \cdot 10 \\
& \sim 0.6 \cdot 10^{4} .
\end{aligned}
$$

The corresponding contribution to $a_{\mu}$,

$$
\delta a_{\mu} \sim 0.6 \cdot 10^{4} \cdot\left(\frac{\alpha}{\pi}\right)^{6} \sim 1 \cdot 10^{-12},
$$

is however way too small to explain even part of the discrepancy.

Could higher order QCD effects be at work? Besides $a_{\mu}^{\mathrm{HVP}-\mathrm{NNLO}}$ [34] already included, higher-order corrections to $a_{\mu}^{\mathrm{HLxL}}$ have also been considered in [52]. The estimated value

$$
a_{\mu}^{\mathrm{HLxL} ; \mathrm{HO}} \sim 0.3(0.2) \cdot 10^{-10},
$$

again fails to match the size of the discrepancy.

Finally, are we already seeing manifestations of BSM degrees of freedom? There are many proposals, see e.g. [53], but the present situation is clearly inconclusive.

Clarification will hopefully come from two new experiments, that are being prepared at FNAL 3, 54 and at J-PARC [55], with the aim of reducing the experimental uncertainty by a factor of 4 . In order to lead to a conclusive situation, these experimental efforts need to be met by a comparable improvement of the theoretical uncertainty. Forthcoming high-precision data from e.g. VEPP-2000, BABAR, BESIII or KLOE-2, should allow for a further reduction of the uncertainty on the hadronic vacuum polarization part, probably below the $0.5 \%$ level. Hadronic light-by-light will then become the dominant source of theoretical errors. There are proposals to address this issue [56 58], but much remains to be done in order to lead these programs to the desired goal. Finally, increasing efforts are being made in order to obtain reliable evaluations of the hadronic contributions to $a_{\mu}$ from QCD simulations on the lattice. The success of this enterprise will hinge on the ability to develop appropriate strategies to overcome the limitations set by the lattice. For interesting proposals and recent advances in this direction, see [59] and references therein, or the contributions in [47], as well as [60] and [61]. This, admittedly incomplete, list of items and of references should merely convey the feeling that the subject remains very active also at the theoretical level. Although the challenge is high, it is reasonable to expect that significant improvements will become available around the time the data from the new experiments will be released.

I would like to close this review with a short remark concerning the anomalous magnetic moment of the electron. Although $a_{e}$ is expected to be less sensitive to new physics than $a_{\mu}$ by a about a factor $\sim\left(m_{\mu} / m_{e}\right)^{2} \sim 40000$, there are exceptions to this naive scaling, and moreover $a_{e}$ is measured with a precision that is $\sim 2300$ times better than the experimental precision on $a_{\mu}$. This leads one to consider the possibility [62] that degrees of freedom beyond the standard model could be first detected in $a_{e}$. Indeed, improvements on both $\Delta a_{e}^{\exp }$ and on the experimental determination of $\alpha$ are within reach [63] on a timescale comparable to the new generation of $(g-2)$ experiments at FNAL and at J-PARC.

We are therefore looking forward to very exciting times in high-precision physics with the forthcoming experiments and, hopefully, with the accompanying improvements on the theoretical aspects.

\section{Acknowledgements}

I would like to thank S. Narison and the Organizing Committee of the QCD-2014 Conference for the invitation to give this presentation. I have benefited from many informative and stimulating discussions with L. Lellouch, A. Nyffeler, and E. de Rafael, and I thank L. Lellouch and E. de Rafael for their comments on the manuscript. I would also like to express my gratitude to the Mainz Institute for Theoretical Physics (MITP) for its hospitality and 
support. This work was supported in part by the OCEVU Labex (ANR-11-LABX-0060) and the A*MIDEX project (ANR-11-IDEX-0001-02) funded by the Investissements dAvenir French government program managed by the ANR.

[1] J. Beringer et al. [Particle Data Group], Phys. Rev. D 86, 010001 (2012).

[2] G. W. Bennett et al., Phys. Rev D 73, 072003 (2006).

[3] B. Lee Roberts, Chin. Phys. C 34, 741 (2010).

[4] D. Hanneke et al., Phys. Rev. Lett. 100, 120801 (2008).

[5] J. Schwinger, Phys. Rev. 73, 416L (1948).

[6] T. Kinoshita (chapter 3), S. Laporta and E. Remiddi (chapter 4) in Lepton Dipole Moments, Advanced Series on Directions in High Energy Physics - Vol. 20, B. Lee Roberts and William J. Marciano Eds, World Scientific Co. Pte. Ltd. (2010).

[7] T. Kinoshita, M. Nio, Phys. Rev. D 73, 053007 (2006). T. Aoyama et al., Phys. Rev. D 78, 053005 (2008); Phys. Rev. D 78, 113006 (2008); Phys. Rev. D 81, 053009 (2010); Phys. Rev. D 82, 113004 (2010); Phys. Rev. D 83, 053002 (2011); Phys. Rev. D 83, 053003 (2011); Phys. Rev. D 84, 053003 (2011); Phys. Rev. D 85, 033007 (2012);

[8] T. Aoyama et al., Phys. Rev. Lett. 109, 111807 (2012).

[9] T. Aoyama et al., Phys. Rev. Lett. 109, 111808 (2012).

[10] P. J. Mohr et al., Rev. Mod. Phys. 84, 1527 (2012).

[11] D. J. Broadhurst et al., Phys. Lett. B 298, 445 (1993).

[12] S. Laporta, Phys. Lett. B 328, 522 (1994).

[13] P. Aguillar et al., Phys. Rev. D 77, 093010 (2008).

[14] P. A. Baikov et al., Nucl. Phys. B, Proc. Suppl. 183, 8 (2008).

[15] A. Kurz et al., Nucl. Phys. B 879, 1 (2014).

[16] R. Bouchendira et al., Phys. Rev. Lett. 106, 080801 (2011).

[17] R. Jackiw, S. Weinberg, Phys. Rev. D 5, 2396 (1972).

[18] I. Bars, M. Yoshimura, Phys. Rev. D 6, 374 (1972).

[19] K. Fujikawa et al., Phys. Rev. D 6, 2923 (1972).

[20] G. Altarelli et al., Phys. Lett. B 40, 415 (1972).

[21] W. A. Bardeen et al., Nucl. Phys. B 46, 315 (1972).

[22] A. Czarnecki et al., Phys. Rev. D 52, 2619 (1995); Phys. Rev. Lett. 76, 3267 (1996).

[23] M. Knecht et al., JHEP11, 003 (2002).

[24] A. Czarnecki et al., Phys. Rev. D 67, 073006 (2003); Err.-ibid. D 73, 119901 (2006).

[25] C. Gnendinger et al., Phys. Rev. D 88, 053005 (2013).

[26] C. Bouchiat, L. Michel, J. Phys. Radium 22, 121 (1961).

[27] L. Durand, Phys. Rev. 128, 441 (1962); Err.-ibid. 129, 2835 (1963).

[28] M. Gourdin, E. de Rafael, Nucl. Phys. B 10, 667 (1969).

[29] M. Davier et al., Eur. Phys. J. C 71, 1515 (2011); Err.-ibid. C 72, 1874 (2012).

[30] K. Hagiwara et al., J. Phys. G 38, 0850003 (2011).

[31] D. Nomura, T. Teubner, Nucl. Phys. B 867, 236 (2013).

[32] R. Barbieri, E. Remiddi, Nucl. Phys. B 90, 233 (1975).

[33] B. Krause, Phys. Lett. B 390, 392 (1997).

[34] A. Kurz et al., B 734, 144 (2014).

[35] M. Knecht, A. Nyffeler, Phys. Rev. D 65, 073034 (2002).

[36] J. Bijnens et al., Phys. Rev. Lett. 75, 1447 (1975) [Err.-ibid. 75, 3781 (1995)]; Nucl. Phys. B 474, 379 (1995); Nucl. Phys. B 626, 410 (2002).

[37] M. Hayakawa et al., Phys. Rev. Lett. 75, 790 (1975); Phys. Rev. D 54, 3137 (1996); Phys. Rev. D 57, 365 (1998) [Err.-ibid. 66, 019902(E) (2002)].

[38] J. Prades, arXiv:hep-ph/0108192.

[39] G 't Hooft, Nucl. Phys. B 72, 461 (1974).

[40] E. Witten, Nucl. Phys. B 160, 57 (1979).

[41] E. de Rafael, Phys. Lett. B 322, 239 (1994).

[42] M. Knecht et al., Phys. Rev. Lett. 88, 071802 (2002)

[43] M. J. Ramsey-Musolf, M. B. Wise, Phys. Rev. Lett. 89, 041601 (2002).

[44] M. J. Savage et al., Phys. Lett. B 291, 481 (1992).

[45] T. Husek et al., arXiv:1405.6927 [hep-ph].

[46] V. Pauk, M. Vanderhaeghen, Eur. Phys. J. C 74, 3008 (2014).

[47] Hadronic contributions to the muon anomalous magnetic moment Workshop. $(g-2)_{\mu}$ : Quo vadis? Workshop. Mini proceedings, arXiv:1407.4021 [hep-ph].

[48] K. Melnikov, A. Vainshtein, Phys. Rev. D 70, 113006 (2004).

[49] A. Nyffeler, Phys. Rev. D 79, 073012 (2009).

[50] J. Prades, E. de Rafael, A. Vainshtein, chapter 9 in Lepton Dipole Moments, quoted in [6].

[51] F. Jegerlehner, A. Nyffeler, Phys. Rept. 477, 1 (2009); A. Nyffeler, Phys. Rev. D 79, 073012 (2009). 
[52] G. Colangelo et al., Phys. Lett. B 735, 90 (2014).

[53] D. Stöckinger, chapter 12 in Lepton Dipole Moments, quoted in [6].

[54] R. M. Carey et al.. The New g-2 experiment - 2009. Fermilab. Proposal 0989.

[55] T. Mibe [J-PARC g-2 Collaboration], Chin. Phys. C 34, 745 (2010).

[56] G. Colangelo et al., JHEP 1409, 091 (2014).

[57] V. Pauk, M. Vanderhaeghen, arXiv:1409.0819 [hep-ph].

[58] G. Colangelo et al., Phys. Lett. B 738, 6 (2014).

[59] T. Blum et al., PoS LATTICE 2012, 022 (2012) arXiv:1301.2607 [hep-lat]].

[60] E. de Rafael, Phys. Lett. B 736, 522 (2014).

[61] T. Blum et al., arXiv:1407.2923 [hep-lat].

[62] G. F. Giudice et al., JHEP 1211, 113 (2012).

[63] F. Terranova, G. M. Tino, Phys. Rev. A 89, 052118 (2014). 